\title{
Serum anti-glycan antibodies in paediatric-onset Crohn's disease: association with disease phenotype and diagnostic accuracy
}

\author{
Małgorzata Sładek ${ }^{1}$, Agata Wasilewska1 ${ }^{1}$ Agnieszka Świat ${ }^{1}$, Adam Ćmiel $^{2}$ \\ 'Department of Paediatrics, Gastroenterology and Nutrition, Jagiellonian University Medical College, Krakow, Poland \\ 2Department of Applied Mathematics, AGH University of Science and Technology, Krakow, Poland
}

Prz Gastroenterol 2014; 9 (4): 232-241

DOI: $10.5114 / p g .2014 .45106$

Key words: inflammatory bowel disease, Crohn's disease, serum anti-glycan antibodies, serological biomarkers, children.

Address for correspondence: Małgorzata Sładek MD, PhD, Department of Pediatrics, Gastroenterology and Nutrition, Jagiellonian University Medical College, 265 Wielicka St, 30-663 Krakow, Poland, phone: +48 1265740 12, fax: +48 1265810 88, e-mail: misladek@cyfronet.pl

\begin{abstract}
Introduction: Antibodies reacting with various microbial epitopes have been described in inflammatory bowel disease (IBD) and are associated with a specific diagnosis and clinical presentation.

Aim: To evaluate the profile of new anti-glycan antibodies, their potential association with disease phenotype and diagnostic accuracy in paediatric Crohn's disease (CD).

Material and methods: Blood samples from 134 paediatric IBD patients (109 CD, 25 ulcerative colitis (UC)) and 67 controls were blindly analysed for anti-Saccharomyces cerevisiae (ASCA), anti-chitobioside carbohydrate (ACCA), anti-laminaribioside carbohydrate (ALCA), and anti-mannobioside carbohydrate (AMCA) antibodies using commercially available assays. The serological response to glycans was correlated with clinical disease characteristics.

Results: At least one of the tested anti-glycan antibodies was present in 75\% of CD patients. Despite the high frequency of reactivity to glycan epitopes, a limited overlap of serological markers was observed. In total, $49 \%$ of ASCA-negative patients presented with one of the following: ACCA, ALCA, or AMCA. The occurrence of one antibody from the anti-glycan panel was independently associated with complicated disease phenotype and ileocolonic disease location. A higher level of immune response as assessed by the quartile sum scores for ACCA, ALCA, and AMCA was linked with older age at diagnosis (10-17 years) and ileocolonic disease location. The ASCA had the greatest accuracy for diagnosis and differentiation of CD.

Conclusions: Qualitative and quantitative serologicalal response to glycan epitopes was associated with distinct clinical presentation in paediatric CD patients. This raises the possibility for the use of these markers to differentiate subgroups of $C D$ patients with more sever clinical presentation. The ASCA was the most accurate serological marker for CD; however, testing for the new anti-glycan antibodies may constitute an adjunctive tool in a specific group of patients to aid in the differentiation of CD with absent ASCA from ulcerative colitis.
\end{abstract}

\section{Introduction}

Crohn's disease (CD) and ulcerative colitis (UC), the two major subtypes of inflammatory bowel disease (IBD), represent heterogeneous groups of chronic, systemic inflammatory disorders, mainly affecting the gastrointestinal tract. Although the exact aetiology still remains a subject of debate, there is clear evidence that an aberrant immune response to intestinal microbiome components affected by environmental factors plays a role in genetically predisposed individuals $[1,2]$. Both UC and CD present with a wide range of pathogenetic pathways that have diverse and often nonspecific clinical presentations, a variable course, and an unpredictable response to treatment [3-6]. Establishing the diagnosis and differentiation of CD and UC is a complex and sometimes challenging process, based on a combination of clinical, laboratory, imaging, endoscopic, and pathological criteria [7, 8]. Despite impressive progress in the evaluation tools, in some patients the diagnosis is delayed or missed, and a subset of patients with isolated colitis remains unclassified. In the data recently published by de Bie et al. on the European paediatric IBD registry, 9\% of 2087 newly diagnosed patients were identified with an unclassified type of the disease (IBD-U) [5]. 
Aberrations in immune response are well known to be present in IBD, and antibodies directed against a variety of microbial and self antigens have been proposed as useful tools in differentiating patients with IBD from non-IBD individuals, and in distinguishing the subtypes of IBD [9-11]. The first described and yet the most extensively studied CD-associated serological marker directed to mannose epitopes of the cell wall of the yeast Saccharomyces cerevisiae (ASCA) is present in 29-69\% of CD patients, $0-29 \%$ of UC subjects, and $0-16 \%$ of healthy controls [9, 10, 12]. For UC, DNase-sensitive "atypical" antibodies directed to perinuclear components of neutrophils (pANCA), present in $41-73 \%$ of UC, $6-38 \%$ of CD subjects, and $0-8 \%$ of controls, remain a recognisable serological marker $[9,10]$. The results presented by numerous studies indicate that both ASCA and PANCA individually have only modest accuracy in detecting and differentiating inflammatory bowel disease, and concerns about the sensitivity of the reported wide range extending from $37 \%$ to $72 \%$ limit their usability as a screening tool $[7,13]$. After pANCA and ASCA, many additional types of antibodies have been described as being linked with CD, including those against Pseudomonas fluorescens-associated sequence 12 (Anti-12), outer membrane porin $\mathrm{C}(\mathrm{OmpC})$ of Escherichia coli, and bacterial flagellin cBir1 (Anti-CBir1) [10, 14]. The correlation of the presence (qualitative) and level of serological (quantitative) response to those various protein and carbohydrate antigens with the disease phenotype, disease severity, and the course of disease and disease-related surgery requirements, have been reported for $C D$ patients $[9,10$, 15-18]. More recently, the presence of members of the anti-glycan antibodies family other than ASCA, including anti-mannobioside carbohydrate IgA antibodies (AMCA), anti-laminaribioside carbohydrate IgA antibodies (ALCA), and anti-chitobioside carbohydrate IgG antibodies (ACCA), has been recognised as being associated with CD in adults, but limited data are available with respect to paediatric patients [19-22].

In the era of personalised medicine, identification of patients with complicated disease and a poor prognosis is becoming a challenging issue, with special importance attached to decision-making strategy to optimise the therapy for individual patients.

\section{Aim}

Therefore, it has been our objective in the present study to assess the profile of serological response to the panel of glycan epitopes (ASCA, ACCA, ALCA, and AMCA) and to evaluate a new set of anti-glycan antibodies, their diagnostic and differential usefulness, and their potential association with disease phenotype in paediatric-onset CD patients.

\section{Material and methods \\ Patient population}

Blood samples were obtained from 109 CD patients and 25 UC patients, who were referred for investigation to our tertiary-care referral centre. As the controls, we recruited 67 children (34 boys, 33 girls, mean age ( \pm SD) $10.63 \pm 4.56$ years) diagnosed with acute diseases (acute gastroenteritis, acute ear, nose, or throat disorders). The exclusion criteria for the controls included the following: suspicion of inflammatory bowel disorders, gastrointestinal bleeding, presence of any chronic diseases, and positive family history for immunological and gastrointestinal disorders. Diagnosis and differentiation of CD and UC were performed based on clinical, endoscopic, histological, and imaging investigations according to the Porto criteria [7]. The phenotype of the disease was assigned using the Paris classification [8]. Clinical and demographic characteristics of the IBD patients are presented in Table I. Informed consent was obtained from all parents/ legal guardians and from patients above 16 years of age. The study was approved by the Institutional Research Ethics Board at the Jagiellonian University Medical College.

\section{Serological analysis}

All blood samples were drawn at the time of the enrolment, and further analysis was performed in a random and blind fashion. To detect ACCA IgA, ALCA IgG, and AMCA the IgG sera were immediately separated by centrifugation and stored at $-20^{\circ} \mathrm{C}$ until analysis, which was performed with commercially available, specific enzyme-immunosorbent assay (ELISA) kits (Glycominds LTD, Lod, Israel) according to the manufacturer's protocol (Glycominds LTD, Lod, Israel). The results were presented as arbitrary ELISA units (EU), which were calculated based on the sample and calibrator optical density and documented both in absolute values and frequency of positivity. The cut-off values for positive tests were assessed according to the manufacturer's recommendations as follows: $90 \mathrm{EU} / \mathrm{ml}, 60 \mathrm{EU} / \mathrm{ml}$, and $100 \mathrm{EU} / \mathrm{ml}$ for ACCA, ALCA, and AMCA, respectively. In addition to the qualitative analysis, to evaluate the level of immune response of ACCA, ALCA, and AMCA and possible association with distinct $C D$ phenotypes, quartile scores for each of these serologicalal markers were calculated. In brief, for each antigen, the patients whose antibody levels were in the first, second, third, and fourth quartile of the distribution were assigned a quartile score of 1, 2, 3, and 4 , respectively. By adding individual quartile scores for each of the glycan antigens a quartile sum score (range Q3-Q12) representing the cumulative immune response toward all the three new anti-glycan antigens for each individual patient was calculated. 
Table I. Characteristics and clinical phenotypes of the inflammatory bowel disease cohort

\begin{tabular}{|c|c|c|}
\hline Variables & $\begin{array}{l}\text { Crohn's disease patients } \\
\qquad(N=109)\end{array}$ & $\begin{array}{l}\text { Ulcerative colitis patients } \\
\qquad(N=25)\end{array}$ \\
\hline Male/female, $n(\%)$ & $64 / 45(58.7)$ & $8 / 17(32.0)$ \\
\hline Median age at diagnosis (IQR) [years] & $11.2(7.4-15.2)$ & $12.8(10.4-15.3)$ \\
\hline Mean age of symptoms onset $( \pm$ SD) [years] & $10.0 \pm 4.8$ & $11.8 \pm 3.5$ \\
\hline Mean time of symptoms duration before diagnosis ( \pm SD) [months] & $12.4 \pm 18.9$ & $3.05 \pm 4.1^{*}$ \\
\hline $\begin{array}{l}\text { CD age }(\mathrm{A}) \text { at diagnosis, } n(\%) \text { : } \\
\text { A1a (<10 years) } \\
\text { A1b (10-17 years) } \\
\text { A2 (17-40 years) }\end{array}$ & $\begin{array}{l}46(42.2) \\
47(43.1) \\
16(14.7)\end{array}$ & $\begin{array}{l}- \\
- \\
-\end{array}$ \\
\hline $\begin{array}{l}\text { CD location (L), } n(\%) \text { : } \\
\text { L1 (ileal) } \\
\text { L2 (colonic) } \\
\text { L3 (ileocolonic) } \\
L 4_{a}+\angle 4_{b} \text { (upper GI proximal to } 1 / 3 \text { distal ileum) }\end{array}$ & $\begin{array}{c}8(7.3) \\
41(37.6) \\
60(55.0) \\
53(48.6)\end{array}$ & $\begin{array}{l}- \\
- \\
- \\
-\end{array}$ \\
\hline $\begin{array}{l}\text { CD behaviour (B), } n(\%) \text { : } \\
\text { B1 (inflammatory) } \\
\text { B2 (stricturing) } \\
\text { B3 (penetrating) } \\
\text { p (perianal disease) }\end{array}$ & $\begin{array}{c}91(83.5) \\
15(13.7) \\
3(2.8) \\
28(25.7)\end{array}$ & $\begin{array}{l}- \\
- \\
- \\
-\end{array}$ \\
\hline $\begin{array}{l}\text { CD growth retardation (G), } n(\%) \text { : } \\
\text { GO (no evidence) } \\
\text { G1 (growth delay) }\end{array}$ & $\begin{array}{l}78(71.6) \\
31(28.4)\end{array}$ & $\begin{array}{l}- \\
-\end{array}$ \\
\hline $\begin{array}{l}\text { UC extension (E), } n(\%): \\
\text { E1 (proctitis) } \\
\text { E2 (distal to splenic flexure) } \\
\text { E3 (distal to hepatic flexure) } \\
\text { E4 (proximal to hepatic flexure) }\end{array}$ & $\begin{array}{l}- \\
- \\
- \\
-\end{array}$ & $\begin{array}{c}1(4.0) \\
2(8.0) \\
3(12.0) \\
19(76.0)\end{array}$ \\
\hline $\begin{array}{l}\text { UC severity (S), } n(\%) \text { : } \\
\text { SO (never sever; PUCAI < 65) } \\
\text { S1 (ever severe; PUCAI } \geq 65 \text { ) }\end{array}$ & - & $\begin{array}{l}17(68.0) \\
8(32.0)\end{array}$ \\
\hline
\end{tabular}

$C D$-Crohn's disease, UC - ulcerative colitis, SD - standard deviation, IQR - interquartile range, PUCAI - Paediatric Ulcerative Colitis Activity Index, ${ }^{*} p<0.001$

Determination of ASCA IgA and IgG was performed by the indirect immunofluorescence technique using a commercially available kit according to the manufacturer's instructions (EUROIMMUN, Germany). The cut-off values for positivity were set at 1/100 for IgG and $1 / 1000$ for IgA, as recommended by the manufacturer (EUROIMMUN, Germany). Diagnostic parameters (sensitivity, specificity, positive and negative predictive values) were calculated individually for each of the serological markers. To determinate the accuracy of ACCA, ALCA, and AMCA in differentiation between CD, UC, and the controls independently of the cut-off values, receiver operating characteristic (ROC) curves were generated by plotting sensitivity versus 1-specificity.

\section{Statistical analysis}

Data were analysed using StatSoft Statistica software system version 10 . All the quantitative variables were presented as means \pm standard deviations (SD) for symmetrically distributed data, or as median interquartile range (INQ) for skewed data. All categorical variables were presented as numbers of patients and percentages. Unpaired categorical data were compared using the $\chi^{2}$ or Fischer's exact test, as appropriate. The unpaired $t$-test, Wilcoxon's rank-sum test, or Kruskal Wallis test were used to compare continuous variables, as appropriate for the data normality and number of contrasting groups. The Mann-Whitney test was used for comparison of medians. Multivariate logistic regression analysis was performed to define independent predictors of seropositivity for each individual marker and age at diagnosis, location, and phenotype of CD.

For the analysis of quantitative immunological response, the Cochran-Armitage test was used to determine whether there was a linear trend between the ordered categories of antibody response defined by quartiles and the proportion of patients with a specific disease. Results were expressed as odds ratios (Odds) 
and $95 \%$ confidence intervals (Cls). Statistical significance was defined as a two-tailed $p$-value $<0.05$.

\section{Results}

\section{Presence and profile of anti-glycan antibodies in Crohn's disease patients}

A significant proportion of $C D$ patients responded to glycan antigens. The prevalence of ASCA, ACCA, ALCA, and AMCA in CD and UC patients as well as the controls is presented in Table II. ASCA were positive in $59.6 \%$ of CD patients and in $10.5 \%$ of the controls (OR $=12.38$, 95\% Cl: 5.19-29.57, $p<0.001)$. There was no difference in the prevalence of ASCA in UC compared to the control group ( $\mathrm{OR}=0.745,95 \% \mathrm{Cl}: 0.144-3.857, p=0.726)$, but it was significantly lower as compared to CD patients (OR $=0.001,95 \% \mathrm{Cl}: 0.0003-0.006, p<0.001)$. Among the new anti-glycan antibodies, ALCA were significantly more prevalent in CD than in UC $(O R=14.614$, $95 \% \mathrm{Cl}: 2.858-240.979, p<0.007)$ or in the controls $(\mathrm{OR}=9.176,95 \% \mathrm{Cl}: 2.092-40.254, p<0.001)$. For ACCA, the values approximated significance for the controls $(\mathrm{OR}=2.078,95 \% \mathrm{Cl}: 0.987-4.375, p=0.055)$ but were not different in comparison to UC patients $(O R=2.380$, 95\% Cl: 0.783-7.470, $p=0.137$ ).

The presence of antibodies directed to one of the glycan epitopes (ASCA, ACCA, ALCA, or AMCA) was significantly associated with CD (Table II). At least one positive antibody of the panel was more prevalent in CD patients compared both to UC subjects $(O R=9.617,95 \% \mathrm{Cl}: 3.482-26.562, p<0.001)$ and the controls $(O R=7.137,95 \% \mathrm{Cl}: 3.614-14.095$, $p=0.001)$. Seventy-five percent of CD patients were positive for one of the anti-glycan antibodies, while $76 \%$ of UC patients and $70 \%$ of the controls did not show any antibodies. A higher level of the immune response as expressed by the number of positive anti-glycan antibodies was significantly associated with CD ( $p=0.0097$ ); however, a limited overlap of antibodies was observed. The sera of $33.9 \%$ of CD patients reacted with two glycan epitopes, while $11.9 \%$ of CD subjects were positive for three antigens and only 1 patient expressed all four anti-glycan antibodies (Table II). Testing for ACCA, ALCA, and AMCA allowed for serological identification of an additional subgroup of CD patients, who did not express immunoreactivity toward mannan epitopes of Saccharomyces cerevisiae. Among 45 ASCA-negative CD patients, serological response to one of the new glycan epitopes was found in almost half (49\%) of them, including ACCA present in 31\%, ALCA in $13 \%$, and $A M C A$ in $4 \%$ of the subjects.

\section{Diagnostic accuracy and use for diagnosis and differentiation}

Based on the manufacturer-recommended cut-off values the sensitivity and specificity, positive and negative predictive values of the serological markers were calculated for clinical situations differentiating CD from the controls and distinguishing CD from UC. Table III shows the results for each of the individual markers. All of the anti-glycan antibodies were highly specific (80-100\%) for CD, with the highest values characterising ALCA (97-100\%). ASCA presented the highest sensitivity for differentiation of CD from both the controls (59\%) and UC (59\%). The sensitivity of the other an-

Table II. Anti-glycan antibodies distribution in the studied groups

\begin{tabular}{|c|c|c|c|}
\hline Factor & $\begin{array}{l}\text { Crohn's disease patients } \\
\qquad(N=109)\end{array}$ & $\begin{array}{l}\text { Ulcerative colitis patients } \\
\qquad(N=25)\end{array}$ & $\begin{array}{l}\text { Controls } \\
(N=67)\end{array}$ \\
\hline \multicolumn{4}{|c|}{ Median (IQR) levels, EU: } \\
\hline ACCA & $37.7(21.5-54.7)$ & $33.2(24.7-40.3)$ & $30.9(23.3-46.1)$ \\
\hline ALCA & $25.8(18.4-57.0)$ & $24.0(19.1-30.7)$ & $25.4(18.8-34.1)$ \\
\hline AMCA & $42.8(31.33-60.2)$ & $37.4(30.1-54.9)$ & $39.1(29.2-59.1)$ \\
\hline \multicolumn{4}{|c|}{ Antibody positivity, n (\%) } \\
\hline ASCA*** & $65(59.6)$ & $2(8)$ & $7(10.5)$ \\
\hline $\operatorname{ACCA}(\geq 90 \mathrm{EU})$ & $34(31.1)$ & $4(16)$ & $12(17.9)$ \\
\hline $\operatorname{ALCA}(\geq 60 \mathrm{EU})^{*, * *}$ & $24(22)$ & $0(0)$ & $2(2.9)$ \\
\hline $\mathrm{AMCA}(\geq 100 \mathrm{EU})$ & $11(10.1)$ & $2(8)$ & $4(5.9)$ \\
\hline \multicolumn{4}{|c|}{ Number of positive AGABs, $n(\%)^{\star, * *}$} \\
\hline 1 & $82(75.2)$ & $6(24)$ & $20(29.9)$ \\
\hline 2 & 37 (33.9) & $2(8)$ & $5(7.5)$ \\
\hline 3 & $13(11.9)$ & $0(0)$ & $0(0)$ \\
\hline 4 & $1(1)$ & $0(0)$ & $0(0)$ \\
\hline
\end{tabular}

$C D$ - Crohn's disease, UC - ulcerative colitis, ACCA - anti-chitobioside carbohydrate antibody, ALCA - anti-laminaribioside carbohydrate antibody, AMCA - anti-mannobioside carbohydrate antibody, ASCA - anti-Saccharomyces cerevisiae antibody, AGABs - anti-glycan antibodies (positive ASCA, ACCA, $A L C A$, or AMCA), IQR - interquartile range, EU - ELISA units, ${ }^{*} p<0.05 C D$ vs. controls, ${ }^{* *} p<0.05 C D$ vs. UC 
Table III. Diagnostic accuracy of each studied marker for differentiation of CD $(n=109)$ with the controls $(n=67)$ and UC $(n=25)$

\begin{tabular}{|c|c|c|c|c|c|c|c|c|}
\hline & $\begin{array}{c}\text { Sensitivity } \\
\text { [\%] }\end{array}$ & 95\% Cl [\%] & $\begin{array}{c}\text { Specificity } \\
{[\%]}\end{array}$ & $95 \%$ Cl [\%] & PPV & $95 \% \mathrm{Cl}$ [\%] & NPV [\%] & $95 \% \mathrm{Cl}$ [\%] \\
\hline \multicolumn{9}{|l|}{ CD vs. controls: } \\
\hline ASCA & 59 & $49-68$ & 90 & $80-96$ & 90 & $81-96$ & 57 & $47-67$ \\
\hline $\mathrm{ACCA}(\geq 90 \mathrm{EU})$ & 31 & $23-41$ & 82 & $71-90$ & 74 & $59-86$ & 42 & $34-51$ \\
\hline $\operatorname{ALCA}(\geq 60 \mathrm{EU})$ & 27 & $18-37$ & 97 & 90-99 & 92 & $75-99$ & 50 & $41-59$ \\
\hline AMCA ( $\geq 100 \mathrm{EU})$ & 10 & $5-17$ & 92 & $74-99$ & 85 & $55-98$ & 19 & $12-27$ \\
\hline \multicolumn{9}{|l|}{ CD vs. UC: } \\
\hline ASCA & 59 & $49-68$ & 92 & $74-99$ & 97 & 90-99 & 34 & $23-46$ \\
\hline $\mathrm{ACCA}(\geq 90 \mathrm{EU})$ & 33 & $24-43$ & 80 & 59-93 & 88 & $74-96$ & 22 & $14-31$ \\
\hline $\operatorname{ALCA}(\geq 60 \mathrm{EU})$ & 22 & $15-31$ & 100 & $86-100$ & 100 & $86-100$ & 23 & $15-32$ \\
\hline $\mathrm{AMCA}(\geq 100 \mathrm{EU})$ & 10 & $5-17$ & 92 & $74-99$ & 85 & $55-98$ & 19 & $12-27$ \\
\hline
\end{tabular}

CD - Crohn's disease, UC - ulcerative colitis, ACCA - anti-chitobioside carbohydrate antibody, ALCA - anti-laminaribioside carbohydrate antibody, AMCA anti-mannobioside carbohydrate antibody, ASCA - anti-Saccharomyces cerevisiae antibody, PPV - positive predictive value, NPV - negative predictive value, Cl-confidence interval, EU - ELISA unit

ti-glycan antibodies was low and had values from 10\% to $33 \%$ (Table III). The best performance for positive predictive values was shown by ALCA (92-100\%) and ASCA (90-97\%). For clinical situations, when negative predictive values are of importance to avoid invasive diagnostic procedures, all the new anti-glycan antibodies presented poor levels that ranged from 19\% to 50\%, as shown in Table III.

To differentiate CD from the controls and UC independently of the cut-off levels, we constructed ROC curves for ACCA, ALCA, and AMCA. All the anti-glycan antibodies showed a weak discriminatory capacity for differentiation of CD from UC $\left(A \cup C_{A C C A}-0.554\right.$, 95\% Cl: 0.445-0.664; $\mathrm{AUC}_{\mathrm{ALCA}}-0.611,95 \% \mathrm{Cl}: 0.506-$ 0.716 ; $\left.A_{U C C_{A M C A}}-0.568,95 \% \mathrm{Cl}: 0.443-0.693\right)$ and the controls (AUC ACCA $-0.5596,95 \% \mathrm{Cl}: 0.474-0.645$; AUC $_{\text {ALCA }}$ $-0.572,95 \% \mathrm{Cl}: 0.488-0.656, \mathrm{AUC}_{\text {AMCA }}-0.553,95 \% \mathrm{Cl}$ : 0.464-0.642). The only significant difference in antibodies level was found for ALCA, which presented higher values in the older (10 to 17 years) age group (46.3 \pm 31.54 vs. $31.9 \pm 24.25 \mathrm{EU}, p=0.012$ ).

\section{Serological response association with disease phenotype}

In the univariate analysis, no association was found between the prevalence of each individual marker and disease phenotype, including age at diagnosis, disease behaviour, and disease location $(p>0.05)$. To determine which antibody was independently associated with specific disease characteristics, multivariate logistic regression analysis was performed. Taking into account separately age at diagnosis, disease localisation, and disease behaviour as potential confounders, we found an inde- pendent association between one of the newer anti-glycan antibodies (ACCA, ALCA, AMCA) and ileocolonic disease location $(\mathrm{L} 3$; OR $=4.444,95 \% \mathrm{Cl}$ : 1.670-11.830, $p=0.0147)$. Among antibodies, only ALCA was significantly more likely to be linked with this disease location $(\mathrm{OR}=4.429,95 \% \mathrm{Cl}: 0.5733-34.211, p=0.018)$. Isolated ileal disease was omitted from this analysis because of the low number of patients with this location. The multivariate logistic regression model revealed that the presence of one antibody of the entire anti-glycan panel (ASCA, ACCA, ALCA, AMCA) was associated with complicated disease behaviour (B2, B3) $(p=0.043)$.

To evaluate a possible association between the level of antibody response to the new anti-glycan antigens and $C D$ phenotype, the quartile scores analysis individually for ACCA, ALCA, and AMCA (Table IV) and cumulative quartile scores of the sum (Figure 1) for all the antibodies were calculated as stated in the Material and methods section. We found that an increasing cumulative response to glycans was associated with older age at diagnosis ( $p=0.0467)$ and a decreasing likelihood for disease localisation limited to the colon $(p=0.0295)$. For individual antibodies, an increasing response associated with older age at diagnosis was significant for $\operatorname{ALCA}(p=0.017)$, and decreasing disease localisation in the colon was significant for ACCA $(p=0.0014)$. There was no trend among the quartile scores for any of the individual antibodies or for the quartile sum scores and disease behaviour $(p>0.05)$.

\section{Discussion}

In the present study of a well-defined paediatric IBD cohort, we evaluated a potential association between 
Table IV. Quartile analysis of the Crohn's disease cohort $(n=109)$ for the three markers (ACCA, ALCA, and AMCA) for age at diagnosis (A), disease behavior (B), and disease location ( $\mathrm{L}$ )

\begin{tabular}{|c|c|c|c|c|c|c|}
\hline Markers & & Q1 & Q2 & Q3 & Q4 & Value of $p$ \\
\hline \multirow[t]{2}{*}{ ACCA } & Ala & 15 (33\%) & 13 (28\%) & $7(15 \%)$ & 11 (24\%) & 0.15 \\
\hline & Alb & $9(19 \%)$ & $11(23 \%)$ & 15 (32\%) & 12 (26\%) & \\
\hline \multirow[t]{2}{*}{ ALCA } & Ala & $16(35 \%)$ & 14 (30\%) & $9(20 \%)$ & $7(15 \%)$ & 0.017 \\
\hline & $A 1 b$ & $9(19 \%)$ & $10(21 \%)$ & 14 (30\%) & 14 (30\%) & \\
\hline \multirow[t]{2}{*}{ AMCA } & Ala & 16 (35\%) & $9(20 \%)$ & 11 (24\%) & 10 (22\%) & 0.380 \\
\hline & Alb & $11(23 \%)$ & $11(23 \%)$ & 14 (30\%) & $11(23 \%)$ & \\
\hline \multirow[t]{2}{*}{ ACCA } & B1 & 24 (26\%) & 19 (21\%) & 24 (26\%) & $24(26 \%)$ & 0.388 \\
\hline & $B 2+B 3$ & $4(22 \%)$ & $8(44 \%)$ & $3(17 \%)$ & $3(17 \%)$ & \\
\hline \multirow[t]{2}{*}{ ALCA } & B1 & $25(27 \%)$ & $23(25 \%)$ & $21(23 \%)$ & 22 (24\%) & 0.329 \\
\hline & $\mathrm{B} 2+\mathrm{B} 3$ & $3(17 \%)$ & $4(22 \%)$ & $6(33 \%)$ & $5(28 \%)$ & \\
\hline \multirow[t]{2}{*}{ AMCA } & B1 & 24 (26\%) & $21(23 \%)$ & $23(25 \%)$ & $23(25 \%)$ & 0.863 \\
\hline & $\mathrm{B} 2+\mathrm{B} 3$ & $4(22 \%)$ & $6(33 \%)$ & $4(22 \%)$ & $4(22 \%)$ & \\
\hline \multirow[t]{3}{*}{ ACCA } & L1 & $3(43 \%)$ & $3(43 \%)$ & $0(0 \%)$ & $1(14 \%)$ & \\
\hline & L2 & 14 (34\%) & $9(22 \%)$ & $11(27 \%)$ & $7(17 \%)$ & 0.001 \\
\hline & L3 & 11 (18\%) & $15(25 \%)$ & $15(25 \%)$ & 19 (32\%) & \\
\hline \multirow[t]{3}{*}{ ALCA } & L1 & $2(29 \%)$ & $4(57 \%)$ & $0(0 \%)$ & $1(14 \%)$ & \\
\hline & L2 & $12(29 \%)$ & $9(22 \%)$ & $13(32 \%)$ & $7(17 \%)$ & 0.119 \\
\hline & L3 & 13 (22\%) & 14 (23\%) & 14 (23\%) & 19 (32\%) & \\
\hline \multirow[t]{3}{*}{ AMCA } & L1 & $3(43 \%)$ & $2(29 \%)$ & $0(0 \%)$ & $2(29 \%)$ & \\
\hline & L2 & 13 (32\%) & 11 (27\%) & $8(20 \%)$ & $9(22 \%)$ & 0.117 \\
\hline & L3 & 12 (20\%) & 14 (23\%) & 18 (30\%) & 16 (27\%) & \\
\hline
\end{tabular}

$C D$-Crohn's disease, Q - quartile, ACCA - anti-chitobioside carbohydrate antibody, ALCA - anti-laminaribioside carbohydrate antibody,

AMCA - anti-mannobioside carbohydrate antibody, ASCA - anti-Saccharomyces cerevisiae antibody, Ala - age of diagnosis $<10$ years, Alb - age of diagnosis 10-17 years, B1 - inflammatory disease, B2 - stricturing disease, B3 - penetrating disease, L1 - ileal disease, L2 - colonic disease, L3 - ileocolonic disease

an adaptive response to the panel of glycan epitopes, including ASCA, ACCA, ALCA, and AMCA, and the clinical presentation of $C D$. Moreover, we tested whether the new markers (ACCA, ALCA, and AMCA) could be helpful in disease differentiation and stratification. We confirmed the association between some anti-glycan antibodies (ACCA, ALCA) and CD, detecting a correlation between the presence of antibodies and complicated disease presentation and ileocolonic disease location. In addition, a higher level of adaptive response was associated with older age at diagnosis and ileocolonic disease. Although newer anti-glycan antibodies were highly specific for CD, their capability as a screening tool for diagnosis of primary CD was limited, and ASCA antibodies remain the most accurate single marker for paediatric $C D$.

\section{Profile of anti-glycan antibodies and association with disease phenotype}

In this study, qualitative and quantitative immune response to glycan epitopes was strongly associated with CD and specific disease phenotype. Notably, the serological response to glycans was complex, and despite a relatively high frequency of antibodies present in CD patients, a limited overlap was found. The observed wide variation in both the number and magnitude of immune responses as expressed by the quartile sum scores to glycans indicates that the degree of reactivity to microbial components is not uniform in all paediatric CD patients. Testing for the new set of antibodies, which allowed for serological identification of an additional subgroup of CD patients, may facilitate the discrimination of indeterminate IBD-phenotype. Forty-nine percent of those who did not show immune reactivity toward mannan epitopes of Saccharomyces cerevisiae were positive for at least one other marker from the panel. A similar distribution of anti-glycan antibodies with respect to the status of the markers as well as diversification of the response to anti-glycan epitopes have been published in studies performed in adults [17, 19, 21, 22]. Rieder et al. reported 40.9\% ASCA-negative 

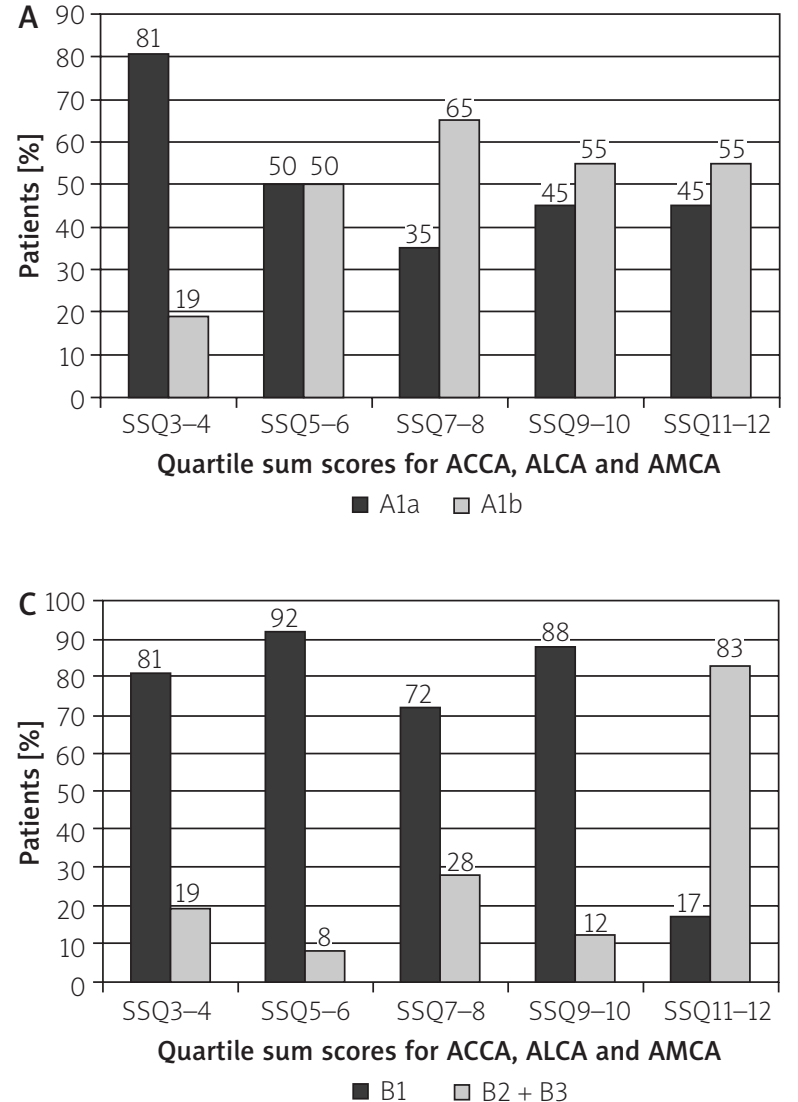

CD children to be positive for at least one other marker from the anti-glycan panel [20]. This illustrates the importance of combined testing of newer glycan antibodies in a specific group of patients, and their potential usefulness as an additional tool in cases of diagnostic uncertainty.

There is a growing body of evidence indicating that the pathogenesis of $C D$ is strongly associated with aberrant recognition of resident intestinal microbial components [1]. Glycans are cell wall surface carbohydrate components that can be found on various pathogenic and saprophytic microorganisms playing a role in diversified immunological processes and stimulating immune response in a Syk-dependent way $[19,23]$. The non-uniform nature of the serological response to glycan epitopes may reflect different levels of the altered host-microbial relationship and might be related to diverse immunological mechanisms (e.g. antigen sampling, antigen recognition, antigen clearance) affected by genetic heterogeneity and/or varied microbiome components [1]. Therefore, specific antibodies may be the sign of an underlying pathomechanism associated with a characteristic disease presentation. The inflammatory behaviour is dominantly diagnosed in CD pa-

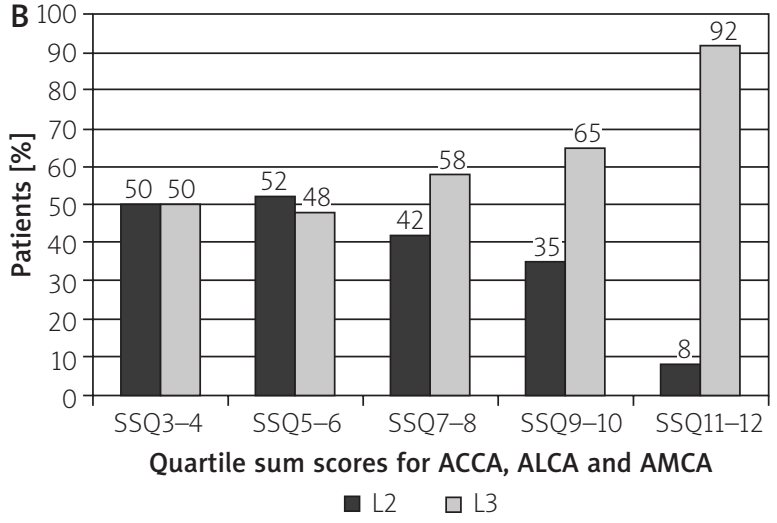

Figure 1. Cumulative quartile sum analysis of the Crohn's disease cohort for the three tested anti-glycan antibodies (ACCA, ALCA, and AMCA). The distribution of quartile sum scores in relation to age at diagnosis $(p=0.47)(\mathrm{A})$, disease localisation $(p=0.047)(B)$ and disease behavior $(p=0.94)(\mathbf{C})$

$C D$-Crohn's disease, SSQ - quartile sum scores (after aggregation by two), ACCA - anti-chitobioside carbohydrate antibody, ALCA - anti-laminaribioside carbohydrate antibody, AMCA - antimannobioside carbohydrate antibody, ASCA - anti-Saccharomyces cerevisiae antibody, Ala - age of diagnosis $<10$ years, A1bage of diagnosis 10-17 years, $L 1$ - ileal localisation, $L 2$ - colonic localisation, L3 - ileocolonic localisation, B1 - inflammatory disease, B2 - stricturing form, B3 - penetrating form

tients at disease onset, but the disease phenotype is not stable and progression to a complicated form can evolve in most patients during years of disease duration $[3,4]$. After initial observations concerning ASCA, several other serologicalal markers have been linked with more complicated disease presentation and severe disease course [15]. Studies in adults indicate that patients with complicated CD disease were more likely to show the presence of anti-glycan antibodies; however, an inconsistent association between individual anti-glycan antibodies and disease phenotype has been reported [17-22, 24-29]. We confirmed that in paediatric patients, anti-glycan antibodies were associated with a complicated disease already at disease onset. Because it was a single time point study with no follow-up, we cannot draw a conclusion addressing a predictive association with the disease course. The only paediatric study to date suggests an association between anti-glycan antibodies and complicated disease phenotype, and a predictive role of the more severe course of the disease [20]. Once the diagnosis of $C D$ is established, anti-glycan antibodies might be an important serologicalal marker for disease stratification and could be considered as an additional tool to identify 
high-risk patients. This may have a significant clinical implication because the introduction of a more potent therapeutic regimen (e.g. biologic and/or immunomodulators) early in the disease course may be of benefit in patients with a higher risk of complicated CD.

In agreement with studies in adults, we found that the magnitude of the immune response to glycans was associated with a more extensive disease localisation, involving the colon and terminal ileum [22, 25]. However, unlike Rieder et al., we were not able to show an association between anti-glycan antibodies and ileal CD [20]. It could be partially explained by the much lower proportion of patients with limited ileal localisation, which in our cohort was present in only 8 (7.3\%) subjects (Table I). Paediatric CD is characterised by a large proportion of colonic involvement, ileitis is usually associated with colitis ( $55 \%$ of the patients in our cohort), and the cumulative probability of isolated ileal CD increases with age until puberty [30]. Differences in disease classification, since we applied the Paris classification and time of blood procurement in relation to the stage of disease duration, may influence the results and limit the possibility of comparing these two studies [8].

\section{Anti-glycan antibodies diagnostic accuracy}

The diagnostic differentiation between CD and UC has both therapeutic and prognostic implications. In the majority of IBD patients it can be made based on clinical, endoscopic, histopathological, and radiological features. However, in a subgroup of patients with strictly colonic disease, differentiation may be a challenge and serological tests could be supportive. As in previous studies, our results indicate that the new anti-glycan antibodies are specific for CD $[9,19]$. However, no antibody demonstrated a sufficient sensitivity to support their usefulness as a screening tool for primary paediatric $C D$ in the diagnostic workup algorithm. The diagnostic accuracy of the ASCA and ALCA for differentiation of CD versus UC has been reported to be similar between adults and children, while some discrepancies have been found for AMCA and ACCA [25, 26, 31]. As in the case of our data, most studies indicate that among the new anti-glycan antibodies, AMCA gives poor values for both sensitivity and specificity [9]. When analysing the differentiation capability of the markers, the new anti-glycan antibodies taken individually have not improved differentiation between CD and UC and the controls. ASCA may still be considered the most accurate single marker from the anti-glycan panel.

Analysis of the diagnostic accuracy of newer anti-glycan antibodies, independently of the recommended cut off values, showed no differences of titre levels between the studied groups. Moreover, the ROC curves have shown a weak discriminatory capability. Data from adult studies are inconsistent in terms of titres for individual markers, as well as a possible association between the serological response and age at the disease onset [20-22, 25, 26]. The only paediatric study indicates that the cut-off values for the new anti-glycan antibodies are lower than those for adults [20]. In our study, an increasing magnitude of the immune response to glycans has been associated with older age at diagnosis, which could be explained by a dependency in antibody production on the age at diagnosis, as previously described [32]. Therefore, the age-dependent differences in serological response should be taken into account and different cut-off values for optimal performance of the anti-glycan antibodies may apply as dependent on age. Further studies on larger patient populations to establish paediatric referral cut-off values are definitely needed.

This study is not without limitations. The first possible limitation is due to a tertiary centre referral bias and higher pre-test probability compared to the general population, due to the diagnosis of CD and UC already having been established at the time of sample procurement. The second limitation is the cross-sectional study design with single point antibody measurements and no longitudinal observation. Therefore, results in the conclusion regarding the prognostic values of the anti-glycan antibodies from this study should be formulated with caution. On the other hand, ASCA appears even years before CD diagnosis, and until now a predominant numbers of studies in adult patients have reported antibody responses to be stable over time, despite different therapeutic modalities being employed, including active and inactive disease [33-35]. As mentioned above, distinct cut-off values for the paediatric CD population, for the optimal performance of the markers, have to be taken into account although the optimal cut-off values for the glycan marker panel have not been determined to date.

\section{Conclusions}

Our results suggest that some new anti-glycan antibodies are associated with paediatric CD and specific disease phenotype, and may be supportive in stratifying disease subtypes. With the growing evidence of an association between serological response and a complicated and more extended disease phenotype, the most important clinical value of anti-glycan antibodies could be in the stratification of patients according to the risk of presentation a more severe disease. Therefore, further studies are definitely needed to evaluate the said risk. The diagnostic significance of individual new anti-glycan antibodies to differentiate IBD from non-IBD 
and CD from UC patients is of limited value and cannot replace conventional clinical evaluation. However, such tests may constitute an adjunctive tool in a specific group of patients, to aid in the differentiation of CD patients with absent ASCA from ulcerative colitis subjects.

\section{Acknowledgments}

The study was supported by Grant K/ZDS/000663.

The authors would like to thank the physicians and study nurses of the Department of Paediatrics, Gastroenterology, and Nutrition for their support of the study, and Barbara Hajto for her excellent technical support and for performing serological assays.

\section{References}

1. Abraham C, Cho JH. Inflammatory bowel disease. N Engl I Med 2009; 361: 2066-78.

2. Kustosz P, Durlik M. Molecular pathogenesis of Crohn's disease [Polish]. Prz Gastroenterol 2013; 8: 21-6.

3. Ordas I, Eckmann L, Talamini M, et al. Ulcerative colitis. Lancet 2012; 380: 1606-19.

4. Baumgart DC, Sandborn WJ. Crohn's disease. Lancet 2012; 380: 1590-605.

5. De Bie Cl, Paerregaard A, Kolacek S, et al. Disease phenotype at diagnosis in pediatric Crohn's disease: 5-year analyses of the EUROKIDS registry. Inflamm Bowel Dis 2013; 19: 378-85.

6. Kierkuś J, Sładek M, Pytrus T, et al. Clinical characteristics of 320 pediatric Crohn's disease patients registered in the nationwide Crohn's disease registry in Poland [Polish]. Prz Gastroenterol 2012; 7: 228-32.

7. IBD Working Group of the European Society for Paediatric Gastroenterology, Hepatology and Nutrition. Inflammatory bowel disease in children and adolescents: Recommendations for diagnosis - the Porto criteria. J Pediatr Gastroenterol Nutr 2005; 41: 1-7.

8. Levine A, Griffiths A, Markowitz J, et al. Pediatric modification of the Montreal classification for inflammatory bowel disease: the Paris classification. Inflamm Bowel Dis 2011; 17: 1314-21.

9. Kaul A, Hutfless S, Liu L, et al. Serum anti-glycan antibody biomarkers for inflammatory bowel disease diagnosis and progression: a systematic review and meta-analysis. Inflamm Bowel Dis 2012; 18: 1872-84.

10. Prideaux L, De Cruz P, Ng SC, et al. Serological antibodies in inflammatory bowel disease: a systematic review. Inflamm Bowe Dis 2012; 18: 1340-55.

11. Vernier G, Sendid B, Poulain D, et al. Relevance of serologic studies in inflammatory bowel disease. Curr Gastroenterol Rep 2004; 6: 482-7.

12. Moniuszko A, Wisniewska A, Rydzewska G. Biomarkers in management of inflammatory bowel disease. Prz Gastroenterol 2013; 8: 275-83.

13. Van Assche G, Dignass A, Bokemeyer B, et al. Second European evidence-based consensus on the diagnosis and management of ulcerative colitis part 3: special situations. J Crohns Colitis 2013; 7: 1-33.

14. Landers CJ, Cohavy O, Misra R, et al. Selected loss of tolerance evidenced by Crohn's disease-associated immune responses to auto - and microbial antigens. Gastroenterology 200; 123: 689-99.

15. Dubinsky MC, Lin YC, Dutridge D, et al. Serum immune responses predict rapid disease progression among children with Crohn's disease: immune responses predict disease progression. Am J Gastroenterol 2006; 101: 360-7.

16. Dubinsky MC, Kugathasan S, Mei L, et al. Increased immune reactivity predicts aggressive complicating Crohn's disease in children. Clin Gastroenterol Hepatol 2008; 6: 1105-11.

17. Ferrante M, Henckaerts L, Joossens $M$, et al. New serological markers in inflammatory bowel disease are associated with complicated disease behavior. Gut 2007; 56: 1394-403.

18. Arnott ID, Landers CJ, Nimmo EJ, et al. Sera-reactivity to microbial components in Crohn's disease is associated with disease severity and progression, but not NOD2/CARD15 genotype. Am J Gastroenterol 2004; 99: 2376-84.

19. Dotan I, Fishman S, Dgani Y, et al. Antibodies against laminaribioside and chitobioside are novel serologic markers in Crohn's disease. Gastroenterology 2006; 131: 366-78.

20. Rieder F, Hahn P, Finsterhoelzl L, et al. Clinical utility of anti-glycan antibodies in pediatric Crohn's disease in comparison with an adult cohort. Inflamm Bowel Dis 2012; 18: 1221-31.

21. Simondi D, Mengozzi G, Betteto S, et al. Antiglycan antibodies as serological markers in the differential diagnosis of inflammatory bowel disease. Inflamm Bowel Dis 2008; 14: 645-51.

22. Seow CH, Stempak JM, Xu W, et al. Novel anti-glycan antibodies related to inflammatory bowel disease diagnosis and phenotype. Am J Gastroenterol 2009; 104: 1426-34.

23. Baram L, Spektor L, Elad H, et al. Interaction between glycans and the immune system: do glycans play a role in Crohn's disease pathogenesis? Immunology. Willey-Blackwell Commerce Place, Ma USA; 2011.

24. Rieder F, Schleder S, Wolf A, et al. Association of the novel serologic anti-glycan antibodies anti-laminarin and anti-chitin with complicated Crohn's disease behavior. Inflamm Bowel Dis 2010; 16: 263-74.

25. Malickova K, Lakatos PL, Bortlik M, et al. Anticarbohydrate antibodies as markers of inflammatory bowel disease in a central European cohort. Eur J Gastroenterol Hepatol 2010; 22: 144-50.

26. Rejchrt S, Drahosova M, Kopacova M, et al. Antilaminaribioside and antichitobioside antibodies in inflammatory bowel disease. Folia Microbiol 2008; 53: 373-6.

27. Rieder F, Schleder S, Wolf A, et al. Serum anti-glycan antibodies predict complicated Crohn's disease behavior: a cohort study. Inflamm Bowel Dis 2010; 16: 1367-75.

28. Amre DK, Lu SE, Costea F, et al. Utility of serological markers in predicting the early occurrence of complications and surgery in pediatric Crohn's disease patients. Am J Gastroenterol 2006; 101: 645-52.

29. Koutroubakis IE, Drygiannakis D, Tsirogianni A, et al. Antiglycan antibodies in Greek patients with inflammatory bowel disease. Dig Dis Sci 2011; 56: 845-52.

30. Meinzer $U$, Idestrom M, Alberti C, et al. Ileal involvement is age dependent in pediatric Crohn's disease. Inflamm Bowel Dis 2005; 11: 639-44.

31. Lakatos PL, Papp M, Rieder F. Serologic antiglycan antibodies in inflammatory bowel disease. Am J Gastroenterol 2011; 106: 406-12. 
32. Markowitz J, Kugathasan S, Dubinsky M, et al. Age of diagnosis influences serologic responses in children with Crohn's disease: a possible clue to etiology? Inflamm Bowel Dis 2009; 15: 714-9.

33. Israeli E, Grotto I, Gilburd B, et al. Anti-saccharomyces cerevisiae and antineutrophil cytoplasmic antibodies as predictors of inflammatory bowel disease. Gut 2005; 54: 1232-6.

34. van Schaik FD, Oldenburg B, Hart AR, et al. Serological markers predict inflammatory bowel disease years before the diagnosis. Gut 2013; 62: 683-8.

35. Eser A, Papay P, Primas C, et al. The impact of intestinal resection on serum levels of anti-saccharomyces cerevisiae antibodies (ASCA) in patients with Crohn's disease. Aliment Pharmacol Ther 2012; 35: 292-9.

Received: 11.12 .2013

Accepted: 20.01 .2014 